\section{Hormones, reproduction and disease in the longest-lived rodent: the naked mole rat}

\author{
Manlio Vinciguerra1,2 \\ 1 Mouse Biology Unit, European Molecular \\ Biology Laboratory (EMBL), Rome, Italy; \\ 2Genome Biology Unit, European \\ Molecular Biology Laboratory (EMBL), \\ Heidelberg, Germany
}

\begin{abstract}
Aging is an inescapable human condition, made of hormonal, physical, and behavioral changes. Age-related illnesses and decline result from the interactions between genes and the environment. Life expectancy dramatically increased in the last century with the improvement in health cares and quality of life in the Western World. Despite these advances, the last 20 years of our life are often accompanied by an increased incidence of hormonalrelated diseases, such as cancer, metabolic, cardiovascular and neurological dysturbances. Aging research recently uncovered many of the molecular pathways involved in nutrients and hormonal regulation, and also involved in organism life span using simple laboratory animal model. However this knowledge did not translate in an ameliorated aging and cure for hormonal diseases in humans. A breakthrough in aging research came from recent studies on the longest-lived rodent [the naked mole rat (NMR)]. NMR are able to reach $\sim 30$ years of age in good health. Because of their longevity and sustained health, NMR are a research model for successful aging. The most striking feature of NMR is perhaps their eusociality. Unique among mammals (eusociality is observed in insects such as bees and ants), NMR are organized in highly cooperative colonies. The aim of this review is to summarize our knowledge on hormonal and reproductive aspects in NMR. Furthermore, their resistance to pathological insults will be discussed.
\end{abstract}

\section{Introduction}

Aging is an inescapable human condition, made of physical (hormonal, biochemical, physiological), psychological and behavioral changes. Age-related illnesses and decline result from the interactions between genes and the environment. Life expectancy dramatically increased in the last century with the improvement in health cares and quality of life in the Western World. ${ }^{1}$ Despite these advances, the last
20 years of our life are often accompanied by an increased incidence of cancer, endocrine, metabolic and cardiovascular diseases. However, on the planet there are a few blue zones, areas characterized by clusters of communities of disability-free people living >100 years, like Okinawa Island in Japan and Sardinia in Italy.2,3 People living in these areas grow old and become centenarians in a good state. The statistics reveal a significantly lower risk of heart attack, cancer and hormonal disorders. ${ }^{4}$ The key to extreme human longevity is believed to be a mix of genetics and environmental factors such as healthy habits and dietary patterns. ${ }^{5-7}$ Aging research recently uncovered many of the molecular pathways involved in organism life span [including growth factors (i.e. insulin, IGF-1 and others), enzymes (i.e. Sirtuins, mTOR and others) and transcription factors (FOXOs and others)], using simple laboratory animal models, such as yeast, mice and flies.8,9 The ongoing challenge for scientists in the aging field is to demonstrate the relevance of these animal studies to human longevity, since although the molecular networks involved in aging and in fine-tuning of hormones and metabolic functions are pretty much conserved in the simple animals models studied, human life is orders of magnitude longer than the one of those species. Monkeys are closer to humans both phylogenetically and in terms of life span. It is known since more than 50 years that monkeys and humans share very similar hormonal responses, immune systems and, more recently, it has been shown a genomes sequence homology between $90 \%$ and $98 \%$, depending on the species. Monkeys live between 15 and 45 years, also depending on the species. Studies on monkeys have the disadvantages that only few equipped laboratories can afford this kind of research and that animal handling is laborious and very difficult. Since a few decades, a new animal model to study aging came to the scene: the naked mole rat (NMR, Heterocephalus glaber). NMR are mouse-sized and they are the longest-lived rodents, being able to reach $\sim 30$ years of age in good health. ${ }^{10}$ Because of their longevity and sustained health, NMR are a research model for successful aging. ${ }^{11}$ Unique among mammals, NMR are eusocial and organized in highly cooperative colonies (Figure 1), similarly to insects such as bees and ants. The aim of this review is to summarize our knowledge on hormonal and reproductive aspects in NMR. In addition, their resistance to pathological insults will be described.

\section{Hormones, social behavior and reproduction}

NMR are highly social rodents that live in large colonies organized with a rigid social and reproductive hierarchy. In this kind of organi-
Correspondence: European Molecular Biology Laboratory (EMBL), Mouse Biology Unit, Campus A. Buzzati-Traverso, Monterotondo-Scalo, 00016, Roma, Italy.

E-mail: manliov@hotmail.com; manlio.vinciguerra@embl.it

Key words: naked mole rats, aging, hormones, stress.

Acknowledgments: I am the recipient of an EIPOD fellowship from the EMBL.

Conflict of interest: the authors report no conflicts of interest.

Received for publication: 8 April 2011. Accepted for publication: 23 april 2011.

This work is licensed under a Creative Commons Attribution 3.0 License (by-nc 3.0).

CCopyright M. Vinciguerra, 2011

Licensee PAGEPress, Italy

Endocrinology Studies 2011; 1:e4

doi:10.4081/es.2011.e4

zation, called eusociality, only one female, the queen, breeds. ${ }^{10}$ Eusociality (literally, good sociality) is a term coined for the highest level of social organization and can be understood as an extreme form of cooperative breeding. ${ }^{12}$ NMR live in colonies of 200-300 individuals and in the wild lead a subterranean lifestyle in warm and arid African regions. NMR live in large mazes, with interconnected burrows functionally divided for nesting, ventilation, food storage and toilet utilization. Most colony members are non reproductive subordinates that work cooperatively to rear the young and maintain the underground burrow system. ${ }^{10}$ NMR have a long period of gestation $(\sim 70$ days): a study using Ultrasound Biomicroscopy and 3D-Ultrasonography following up the development of the embryos and of several organs speculated a link between the long pregnancy period, eusociality and longevity in these animals. ${ }^{13}$ Faulkes and colleagues showed a couple of decades ago that non breeding males and breeding males display significant differences in the levels of gonadal hormones, which reflect also in a decreased number and motility of spermatozoa.14-16 Pheromones are not involved in the social suppression or reproduction in males and females NMR. ${ }^{17}$ Experimental removal of breeding males allowed understanding that dominance rank was strongly correlated with body weight, age, and urinary testosterone titres in males. ${ }^{18}$ Breeding males were found to be among the heaviest and oldest males in their respective colonies. In contrast to females, NMR males show no evidence of competition over breeding status. ${ }^{18}$ Eusociality may be correlated with a 
relative lack of sex differences and a reduced influence of gonadal hormones (androgens, estrogens) on some functions to which these hormones are usually tightly linked.19,20 Changes in behavior, in brain anatomy and even in female body morphology accompany changes in social and reproductive status in NMR, ${ }^{20-22}$ and this may be dependent or not of gonadal hormonal changes. ${ }^{19,22-24}$

Members of a NMR colony work cooperatively and sleep together in large huddles. They exhibit very low levels of aggression to other colony members, yet are fiercely xenophobic and will mount vigorous attacks against intruders in the laboratory setting. ${ }^{2}$ Recent biochemical and morphological studies explored the neurobiological and hormonal basis of the complex sociality exhibited by NMR. The distribution pattern of vasopressin (a neuropeptide involved in social recognition memory, i.e. the ability to remember a previously-encountered conspecic, at the base of all mammalian social relationships ${ }^{25}$ ) immunoreactivity in the dorsomedial septum of the brain of NMR, ${ }^{26}$ suggests that his could be related to social recognition memory. The neuropeptide oxytocin, which also modulates social bonding and other social behaviors in many vertebrates, was found to have a localization pattern in the central nervous system on NMR subordinates similar to other species. ${ }^{27}$ However, in NMR subordinates high levels of oxytocin immunoreactivity were found in the nucleus accumbens and in the pre-optic area, region that in other rodents are linked to maternal behavior, ${ }^{27,28}$ indicating that although only the queen gives birth or nurses pups in a NMR colony, most individuals might actively participate in pup care.

\section{Metabolism}

There is a relative paucity of information on NMR metabolism; however several peculiarities came out from performed analyses. Metabolism is typically defined as the set of chemical reactions that happen in living an organism to maintain life. These processes allow organisms to grow and maintain their structures (lipids, protein and also nucleic acids), and respond to their environments. Taking into consideration lipids, a recent study using mass-spectrometry shotgun in mice and NMR lipidomics identified phospholipid molecules [ (in particular those containing docosahexaenoic acid (DHA)] from the skeletal muscle, heart, liver and liver mitochondria. ${ }^{29}$ Phospholipids containing DHA are particularly prone to peroxidation and membrane composition may therefore influence longevity. DHAcontaining phospholipids were found to represent $27-57 \%$ of all phospholipids in mice but
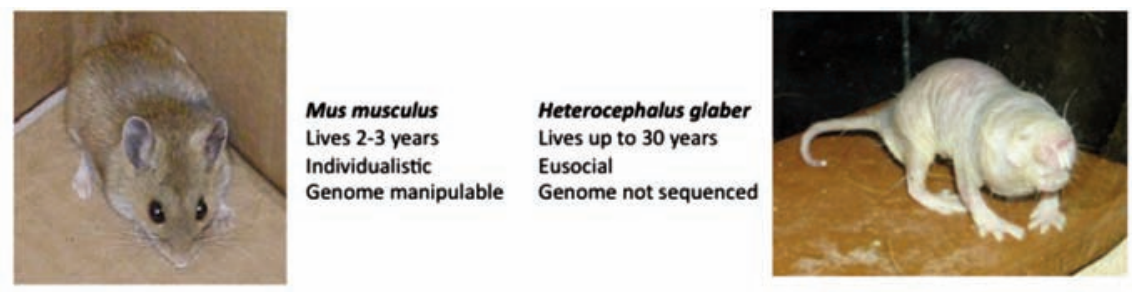

Figure 1. Schematic illustration of the differences between mice and naked mole rat models.

only 2-6\% in NMR. The lower level of DHA-containing phospholipids suggested a lower susceptibility to peroxidative damage in membranes of NMR compared to mice and may contribute to their exceptional longevity. ${ }^{29,30}$ Notwithstanding, comparing age-related profiles of whole-organism lipid peroxidation (urinary isoprostanes) and liver lipid damage (malondialdehyde) in NMR versus mice failed to identify lower levels of lipid peroxidation in NMR, and lipid damage levels did not change with age in mole-rats, suggesting that the enhanced longevity of naked mole-rats is independent of oxidative stress. ${ }^{31}$

An important energy substrate for wholebody and cellular metabolism are the carbohydrates (sugar). Circulating blood sugar levels in mammals are regulated by pancreas-secreted hormones insulin and glucagon. NMR, living in a subterranean environment, has a typical mammalian cold-acclimation response by substantially increasing food intake to meet higher energy requirements when housed at lower temperatures. However, unique among mammals NMR are poikilotherms, which means that their internal temperature varies considerably. Low thyroid hormone concentrations may help to explain this thermoregulatory mode. ${ }^{32}$ Intriguingly, an immunocytochemical and ultrastructural study of NMR pancreas showed that the alpha (glucagon-producing) and beta (insulin-producing) distribution differed from that of other rodents and of humans, in that beta cells formed the mantle while alpha cells formed the internal core of the islets. ${ }^{33}$ Moreover, strange enough NMR are insulin insensitive and they have abnormal responses to glucose tolerance tests. ${ }^{33}$ Insulin-producing cells, however, were more abundant than glucagon-producing cells. ${ }^{33}$ Changes in metabolism and body composition typically occur with aging. Investigations of basal metabolic rate (BMR), body composition and other parameters in 1, 5, 10 and 20-year-old male individuals revealed that among the 5,10 , and 20 -year-olds, there were no age-related changes in body mass, BMR, percentage body fat, fat-free mass or bone mineral density. ${ }^{4}$ Peculiarly, although NMR have low metabolic rates, they have a lifetime energy expenditure more than 4 times that of mice, possibly related also to the fact that they are poikilotherms and live in the wild in a subterranean cold environment compared to the surface. Of note, an analysis of the factors influencing metabolic rate (such as ambient temperature and chronic food restriction) suggested that NMR are capable of long-lasting metabolic adaptations as a means to cope with restricted food supply. ${ }^{35}$ Interestingly, metabolic rate and locomotor activity in NMR were found to follow synchronously a circadian rhythm, suggesting that the circadian system may have significant physiological functions in this subterranean rodent that is not normally exposed to sunlight. ${ }^{36}$ Altogether, the observations exposed in this section demonstrate that, with their unusual thermoregulatory and metabolic features, NMR provide an interesting model for studies of mammalian metabolic regulatory mechanisms.

\section{Stress resistance, disease sus- ceptibility and aging}

According to widely accepted free radical theory of aging postulated in the 50's, the accumulation of free radicals/reactive oxygen species underlies the aging process and disease susceptibility in the organism. ${ }^{37}$ Given the great lifespan of NMR, few reports addressed this issue. Studies of oxidation states of protein cysteines in liver from young and old and NMR showed that age-related changes in protein structural stability, oxidation, and degradation are abrogated over the lifespan of the NMR, indicating that these animals have markedly attenuated age-related accrual of protein oxidation or carbonylation damage and age-associated up-regulation of homeostatic proteolytic mechanisms. ${ }^{38,39}$ These differences suggest that maintenance of protein stability and integrity is crucial to successful aging. Andziak reported that antioxidant defenses of NMR compared to mice did not display any change with age. ${ }^{40} \mathrm{Cu} / \mathrm{Zn}$ superoxide dismutase (Cu/Zn, SOD), Mn SOD, catalase and cellular glutathione peroxidase (cGPx) were measured in livers of physiologically equivalent age-matched NMR and mice. Whereas in mice changes were observed during aging (Mn SOD activity increased with age, while the activity of catalase and cGPx declined), none of the antioxidants changed with age in mole-rats, suggesting that, as also 
mentioned above, it is unlikely that antioxidant defenses are responsible for the longevity difference between these two species.39,40 Intriguingly, a study based on high throughput sequencing approaches has shown that cGPx levels in the liver and kidneys of NMR are an order of magnitude lower than in mice, ${ }^{41}$ which remarkably correlated with a globally reduced utilization of selenium. ${ }^{41}$ Selenium is an essential element in mammals whose supplementation is beneficial for the prevention of several pathologies: it is currently inexplicable how an evolutionary ancient species as NMR has survived with low selenium levels.

It is well established that oxidative stress enhances apoptotic cell death and aging in the blood vessels in part by decreasing nitric oxide (NO) bioavailability. When comparing interspecies differences in endothelial function between mice and NMR, Labinskyy found that sensitivity to acetylcholine-induced, NO-mediated relaxation was smaller in carotid arteries from NMR than in mouse vessels. ${ }^{42}$ In contrast, measurements of production of $\mathrm{O}_{2}^{-*}$ and $\mathrm{H}_{2} \mathrm{O}_{2}$ showed that free radical production in vascular endothelial and smooth muscle cells was comparable in vessels of NMR versus mice. However, whereas in mouse arteries low doses of $\mathrm{H}_{2} \mathrm{O}_{2}$ enhanced apoptotic cell death, in NMR vessels only the highest doses of $\mathrm{H}_{2} \mathrm{O}_{2}$ enhanced apoptotic cell death. ${ }^{42}$ Consistently, in a study comparing NMR and rats, Csiszar showed that whereas rats exhibit a significant age-dependent decline in acetylcholineinduced responses in carotid arteries over a 2yr age range, over a 10 -yr age range nitric oxide (NO)-mediated relaxation responses to acetylcholine were unaltered in NMR.43 In addition, $\mathrm{O}_{2}^{-*}$ and $\mathrm{H}_{2} \mathrm{O}_{2}$ production significantly increased with age in rat arteries, whereas they did not change substantially with age in NMR vessels. ${ }^{43}$ Indicators of apoptotic cell death were 3 -fold enhanced in arteries of 2 -yrold rats; vessels from 12-yr-old NMRs exhibited only a 0.5 fold increase in apoptotic cell death. ${ }^{43}$ Therefore vascular production of reactive oxygen species do not correlate with lifespan, whereas increased lifespan potential of NMR versus short-lived rodents (mice and rats) is associated with an increased vascular resistance to pro-apoptotic stimuli. ${ }^{42,43}$ Oxidative stress in inextricably linked to the pathogenesis of cancer, which is a leading cause of death worldwide. NMR received a lot of attention for their remarkable resistance to cancer. None of the autopsies of dead animals in several colonies have ever revealed the presence of any kind of tumors, ${ }^{44}$ which boosted research efforts from many labs to understand the mechanism underlying this resistance, in the hope of translating eventual findings to humans. Compared to mouse cells, fibroblasts from NMR were found to be resistant to a variety of toxic and destabilizing agents (cadmi- um, methyl methanesulfonate, paraquat, heat, and low-glucose medium), consistent with the fact that cellular resistance to stress may contribute to systemic disease and cancer resistance. ${ }^{45}$ Consistently, transduction of NMR fibroblasts with a combination of two oncogenes, Ras and SV40 LT, fails to induce anchorage-independent growth, while it promptly transforms mouse fibroblasts into a cancerous phenotype. ${ }^{46}$ Only co-expression of a retrovirus encoding hTERT (telomerase reverse transcriptase) could make NMR fibroblasts acquire a malignant phenotype. ${ }^{46}$ In an attempt to explore the mechanisms responsible for the cancer resistance of NMR, Seluanov could demonstrate that NMR fibroblasts display hypersensitivity to contact inhibition, ${ }^{47}$ a chief antitumoral mechanism that arrests cell division when they reach a high density: in culture, NMR fibroblasts arrest at a much lower density compared to mice fibroblasts. Contact inhibition required the activity of p53 and pRb tumor suppressors, ${ }^{47}$ since inactivation of both p53 and pRb attenuated the phenomenon. While contact inhibition in human and mouse is normally triggered by $\mathrm{p} 27$ (Kipl) induction, in NMR was associated with the induction of p16(Ink4a) in an earlier phase, and with the induction of $\mathrm{p} 27$ (Kipl) in a later phase. ${ }^{47}$ Altogether these few reports demonstrated that a special setting and cross-talk of the evolutionary conserved oncogenes and tumor suppressors in NMR cells might underlie the remarkable tumor resistance in this species. Further work needs to be done to shed light on these signaling and protective mechanisms.

Another fascinating stress-related and unique feature of NMR is their sensitivity to pain. In mammals, tissue inflammation leads to pain and behavioral sensitization to thermal and mechanical stimuli. In NMR, signal transducing pain-related neuropeptides (substance P) are absent in cutaneous sensory fibers. ${ }^{48-50}$ NMR show a unique lack of pain-related behaviors to algogens such as capsaicin. The activation of capsaicin-sensitive sensory neurons in naked mole-rats does not produce pain-related behavior, and has anatomical peculiarities. ${ }^{48-50}$ Recently, it has been proposed that the lack of substance P might be the result of adaptation to living in a chronically high carbon dioxide and ammonia environment.51 The pain biology of the NMR is unique among mammals, thus the study of its mechanisms in this unusual species can provide major insights into pain research.

\section{Conclusions and Perspectives}

Although NMR are mouse-sized rodents and relatively easy to grow in laboratories, they are strikingly different from mice due to their social behavior and life span (Figure 1). Decades of studies suggested that NMR are a unique and useful model to study the molecular mechanisms that underlie or protect from metabolic disease and aging. A current obstacle, which is about to be overcome by joint efforts from European and US research groups, is the lack of knowledge of NMR genome full sequence, a factor that presently hampers genetic studies and the development of tools to manipulate the genome as it is feasible in mice, such as knockdown, knock-out or transgenic over-expression of specific genes. ${ }^{52}$ The hope is that the already acquired and the future knowledge on NMR biology could be translated in effective strategies to ameliorate the functional decline associate with human aging.

\section{References}

1. World Health Organization. Report. 2010. Available from: http://www.who.int/ whosis/whostat/EN_WHS10_Full.pdf)

2. Appel LJ. Dietary patterns and longevity: expanding the blue zones. Circulation 2008;118:214-5.

3. Buettner D. The Blue Zone: Lessons for Living Longer From the People Who've Lived the Longest. Washington, DC: National Geographic Society; 2008.

4. Evert J, Lawler E, Bogan H, Perls T. Morbidity profiles of centenarians: survivors, delayers, and escapers. J Gerontol A Biol Sci Med Sci. 2003;58:232-7.

5. Sebastiani P, Solovieff N, Puca A, et al. Genetic Signatures of Exceptional Longevity in Humans. Science 2010 Nov 11. [Epub ahead of print]

6. Newman AB, Glynn NW, Taylor CA, et al. Health and function of participants in the Long Life Family Study: A comparison with other cohorts. Aging (Albany NY) 2011;3:6376 .

7. Heilbronn LK, Ravussin E. Calorie restriction and aging: review of the literature and implications for studies in humans. Am J Clin Nutr 2003;78:361-9.

8. Kenyon CJ. The genetics of ageing. Nature 2010;464:504-12.

9. Chen YF, Wu CY, Kao CH, Tsai TF. Longevity and lifespan control in mammals: lessons from the mouse. Ageing Res Rev 2010;9:S2835 .

10. Edrey YH, Park TJ, Kang H, et al. Endocrine function and neurobiology of the longest-living rodent, the naked mole-rat. Exp Gerontol 2010;46:116-23.

11. Buffenstein R. Negligible senescence in the longest living rodent, the naked mole-rat: insights from a successfully aging species. J Comp Physiol B 2008;178:439-45.

12. O'Riain MJ, Jarvis JU, Alexander R, et al. Morphological castes in a vertebrate. Proc 
Natl Acad Sci U S A 2000;97:13194-7.

13. Roellig K, Drews B, Goeritz F, Hildebrandt TB. The Long Gestation of the Small Naked Mole-Rat (Heterocephalus glaber RUPPELL, 1842) Studied with Ultrasound Biomicroscopy and 3D-Ultrasonography. PLoS ONE 2011;6:e17744.

14. Faulkes CG, Abbott DH. Social control of reproduction in breeding and non-breeding male naked mole-rats (Heterocephalus glaber). J Reprod Fertil 1991;93:427-35.

15. Faulkes CG, Abbott DH, Jarvis JU. Social suppression of reproduction in male naked mole-rats, Heterocephalus glaber. J Reprod Fertil 1991;91:593-604.

16. Faulkes CG, Trowell SN, Jarvis JU, Bennett $\mathrm{NC}$. Investigation of numbers and motility of spermatozoa in reproductively active and socially suppressed males of two eusocial African mole-rats, the naked mole-rat (Heterocephalus glaber) and the Damaraland mole-rat (Cryptomys damarensis). J Reprod Fertil 1994;100: 411-6.

17. Faulkes CG, Abbott DH. Evidence that primer pheromones do not cause social suppression of reproduction in male and female naked mole-rats (Heterocephalus glaber). J Reprod Fertil 1993;99:225-30.

18. Clarke FM, Faulkes CG. Hormonal and behavioural correlates of male dominance and reproductive status in captive colonies of the naked mole-rat, Heterocephalus glaber. Proc Biol Sci 1998;265:1391-9.

19. Holmes MM, Goldman BD, Goldman SL, et al. Neuroendocrinology and sexual differentiation in eusocial mammals. Front Neuroendocrinol 2009;30:519-33.

20. Seney ML, Kelly DA, Goldman BD, et al. Social structure predicts genital morphology in African mole-rats. PLoS ONE 2009;4:e7477.

21. Holmes MM, Rosen GJ, Jordan CL, et al. Social control of brain morphology in a eusocial mammal. Proc Natl Acad Sci USA 2007; 104:10548-52.

22. Holmes MM, Seney ML, Goldman BD, Forger NG. Social and hormonal triggers of neural plasticity in naked mole-rats. Behav Brain Res 2011;218:234-9.

23. Holmes MM, Goldman BD, Forger NG. Social status and sex independently influence androgen receptor expression in the eusocial naked mole-rat brain. Horm Behav 2008;54:278-85.

24. Goldman SL, Forger NG, Goldman BD. Influence of gonadal sex hormones on behavioral components of the reproductive hierarchy in naked mole-rats. Horm Behav 2006;50:77-84.

25. Bielsky IF, Young LJ. Oxytocin, vasopressin, and social recognition in mammals. Peptides 2004;25:1565-74.

26. Rosen GJ, De Vries GJ, Goldman SL, et al. Distribution of vasopressin in the brain of the eusocial naked mole-rat. J Comp Neurol. 2007;500:1093-105.

27. Rosen GJ, de Vries GJ, Goldman SL, et al. Distribution of oxytocin in the brain of a eusocial rodent. Neuroscience 2008;155:80917.

28. Kalamatianos T, Faulkes CG, Oosthuizen MK, et al. Telencephalic binding sites for oxytocin and social organization: a comparative study of eusocial naked mole-rats and solitary cape mole-rats. J Comp Neurol 2010;518:1792-813.

29. Mitchell TW, Buffenstein R, Hulbert AJ. Membrane phospholipid composition may contribute to exceptional longevity of the naked mole-rat (Heterocephalus glaber): a comparative study using shotgun lipidomics. Exp Gerontol 2007;42:1053-62.

30. Hulbert AJ, Faulks SC, Buffenstein R. Oxidation-resistant membrane phospholipids can explain longevity differences among the longest-living rodents and similarly-sized mice. J Gerontol A Biol Sci Med Sci 2006;61:1009-18.

31. Andziak B, Buffenstein R. Disparate patterns of age-related changes in lipid peroxidation in long-lived naked mole-rats and shorterlived mice. Aging Cell 2006;5:525-32.

32. Buffenstein R, Woodley R, Thomadakis C, et al. Cold-induced changes in thyroid function in a poikilothermic mammal, the naked mole-rat. Am J Physiol Regul Integr Comp Physiol. 2001;280:R149-55.

33. Kramer B, Buffenstein R. The pancreas of the naked mole-rat (Heterocephalus glaber): an ultrastructural and immunocytochemical study of the endocrine component of thermoneutral and cold acclimated animals. Gen Comp Endocrinol 2004;139:206-14.

34. O'Connor TP, Lee A, Jarvis JU, Buffenstein R. Prolonged longevity in naked mole-rats: age-related changes in metabolism, body composition and gastrointestinal function. Comp Biochem Physiol A Mol Integr Physiol 2002;133:835-42.

35. Goldman BD, Goldman SL, Lanz T, et al. Factors influencing metabolic rate in naked mole-rats (Heterocephalus glaber). Physiol Behav 1999;66:447-59.

36. Riccio AP, Goldman BD. Circadian rhythms of body temperature and metabolic rate in naked mole-rats. Physiol Behav 2000;71:1522.

37. Harman D. Aging: a theory based on free radical and radiation chemistry. J Gerontol 1956;11:298-300.

38. Perez VI, Buffenstein R, Masamsetti V, et al. Protein stability and resistance to oxidative stress are determinants of longevity in the longest-living rodent, the naked mole-rat. Proc Natl Acad Sci U S A 2009;106:3059-64.

39. Andziak B, O'Connor TP, Qi W, et al. High oxidative damage levels in the longest-living rodent, the naked mole-rat. Aging Cell
2006;5:463-71.

40. Andziak B, O'Connor TP, Buffenstein R. Antioxidants do not explain the disparate longevity between mice and the longest-living rodent, the naked mole-rat. Mech Ageing Dev 2005;126:1206-12.

41. Kasaikina MV, Lobanov AV, Malinouski MY, et al. Reduced utilization of selenium by naked mole rats due to a specific defect in GPx1 expression. J Biol Chem 2011;286: 17005-14.

42. Labinskyy N, Csiszar A, Orosz Z, et al. Comparison of endothelial function, 02-* and $\mathrm{H} 2 \mathrm{O} 2$ production, and vascular oxidative stress resistance between the longest-living rodent, the naked mole rat, and mice. Am J Physiol Heart Circ Physiol 2006;291:H2698704.

43. Csiszar A, Labinskyy N, Orosz Z, et al. Vascular aging in the longest-living rodent, the naked mole rat. Am J Physiol Heart Circ Physiol 2007;293:H919-27.

44. Buffenstein R. The naked mole-rat: a new long-living model for human aging research. J Gerontol A Biol Sci Med Sci 2005;60:136977.

45. Salmon AB, Sadighi Akha AA, Buffenstein R, Miller RA. Fibroblasts from naked mole-rats are resistant to multiple forms of cell injury, but sensitive to peroxide, ultraviolet light, and endoplasmic reticulum stress. J Gerontol A Biol Sci Med Sci 2008;63:232-41.

46. Liang S, Mele J, Wu Y, et al. Resistance to experimental tumorigenesis in cells of a long-lived mammal, the naked mole-rat (Heterocephalus glaber). Aging Cell 2010; 9:626-35.

47. Seluanov A, Hine C, Azpurua J, et al. Hypersensitivity to contact inhibition provides a clue to cancer resistance of naked mole-rat. Proc Natl Acad Sci USA 2009;106: 19352-7.

48. Park TJ, Lu Y, Juttner R, et al. Selective inflammatory pain insensitivity in the African naked mole-rat (Heterocephalus glaber). PloS Biol 2008;6:e13.

49. Smith ES, Blass GR, Lewin GR, Park TJ. Absence of histamine-induced itch in the African naked mole-rat and "rescue" by Substance P. Mol Pain 2010;6:29.

50. Borges RM. Of pungency, pain, and naked mole rats: chili peppers revisited. J Biosci 2009;34:349-51.

51. Brand A, Smith ES, Lewin GR, Park TJ. Functional neurokinin and NMDA receptor activity in an animal naturally lacking substance P: the naked mole-rat. PLoS ONE 2010;5:e15162.

52. van der Weyden L, Adams, DJ, Bradley, A. Tools for targeted manipulation of the mouse genome. Physiol Genomics 2003;11: $133-64$. 